\title{
Las revistas científicas periféricas latinoamericanas ante nuevos escenarios
}

\author{
The peripheral Latin-American scientific journals in the new context
}

\author{
Apolinar Sánchez Hernández (1), Arturo Delgado Sánchez (2) y Violeta Soria Ramírez (3) \\ (1) Departamento de Publicaciones Periódicas de la "Biblioteca Central" DGB-UNAM, Edificio "Biblioteca \\ Central”6 Piso, Ciudad Universitaria, 04510 México, D.F., asanchez@dgb.unam.mx, (2) Centro de \\ Información del Partido Alternativa Social, Av. Insurgentes Sur 1943, Col. Florida, México, D.F. \\ ocelomitl@yahoo.com.mx, (3) Departamento de Consulta de la "Biblioteca Central" DGB-UNAM, Edificio \\ "Biblioteca Central" Entrepiso, Ciudad Universitaria, 04510 México, D.F. miangeldo@yahoo.com.mx
}

\begin{abstract}
Resumen
El presente documento pretende explicar la presencia de las denominadas revistas científicas periféricas latinoamericanas, a partir de un análisis que proporciona la cobertura de aspectos institucionales, técnicos y del impulso para la utilización de tecnologías de información y comunicación en atención a la problemática existente en las revistas aludidas, tomando como muestra a cinco países latinoamericanos en seis áreas del conocimiento específicas. Asimismo, formula una serie de conclusiones y recomendaciones factibles de considerarse como posibles soluciones al fenómeno abordado.
\end{abstract}

Palabras clave: Revistas científicas. Revistas científicas periféricas. Latinoamérica. Tecnologías de Información y Comunicación. Políticas de información científica.

\section{Introducción}

De acuerdo con Allende (1995), la participación activa de un país en el proceso de globalización económica y los intercambios múltiples que suponen los tratados internacionales, exigen, para ser efectuados sobre la base de eficiencia y la competitividad, un incremento de la productividad que no puede lograrse sin los conocimientos científicos generados en los centros de investigación.

Por lo mismo, abundar en la actividad científica representa ingresar a una serie de aspectos en donde destaca la comunicación desarrollada entre los creadores del conocimiento científico; y es en este proceso donde las revistas científicas adquieren un sitio protagónico ineludible.

Para el caso de Latinoamérica - $y$ de otros continentes con similares características- se identifica la existencia de las revistas científicas periféricas que no siempre circulan a nivel re-

\begin{abstract}
The present document tries to explain the presence of the denominated Latin American peripheral scientific journals, from an analysis that provides the cover of institutional, technical aspects and of the impulse for the use of information technologies and communication in the alluded journals, taking as a sample five Latin American countries in six specific areas of knowledge. Also, it formulates a series of conclusions and feasible recommendations for possible solutions.
\end{abstract}

Keywords: Scientific journals. Latin American peripheral scientific journals. Information and Communication Technology. Scientific information policy.

gional, y el desconocimiento de su existencia se vuelve contundente a nivel internacional. Así, cuando se habla de una globalización de la información y de los flujos que esta permite, tal pareciera entonces que dicho flujo no da cobertura para la integración de revistas científicas periféricas, y en tal sentido, la integración de la información científica se ve en cierto modo entorpecida. Estas limitaciones pueden encontrar varias aristas para su análisis que van desde el orden técnico, económico, político y social entre otros factores representativos- que repercuten pero que igualmente pueden dar explicación sobre la marginalidad de las publicaciones aludidas.

Conforme a esta problemática detectada, aparecen las siguientes preguntas centrales para la presente investigación: ¿cuáles son los factores que favorecen y/o entorpecen la integración de las revistas científicas periféricas de Latinoamérica dentro de un contexto científico internacio- 
nal? y ¿cuáles son las tendencias y los escenarios inmediatos y a futuro a los que se enfrentan este tipo de revistas con relación a su integración en el entorno científico internacional?.

Para dar respuesta a estas interrogantes se formulan las siguientes hipótesis: a) el desarrollo económico, científico y cultural en Latinoamérica se encuentra directamente relacionado con el impulso institucional de la región para un reconocimiento científico a nivel internacional y la aportación de las revistas científicas periféricas al contexto mundial; b) la integración y/o marginación de las revistas científicas latinoamericanas al contexto científico internacional depende de la tradición científica de las disciplinas en la región, del intercambio académico con países desarrollados científicamente en áreas específicas y a los criterios de evaluación de la producción científica establecidos en la región y, a su vez, en las de orden internacional.

El objetivo al que se intenta dar cobertura en la presente investigación es identificar y analizar los factores que expliquen la situación de las revistas científicas periféricas latinoamericanas en el contexto regional e internacional, y determinar las tendencias derivadas del uso de las nuevas tecnologías de información y comunicación para el posicionamiento de las revistas científicas periféricas latinoamericanas en el ámbito internacional.

Metodológicamente hablando, y por la naturaleza de nuestro objeto de estudio, se ha convenido utilizar el método deductivo. En este mismo orden, para el análisis formulado se propone un método comparativo, así como una técnica cuantitativa fundamentada en un análisis estadístico-descriptivo - sin invalidar el aspecto cualitativo- que comprende las revistas científicas periféricas latinoamericanas de los cinco países latinoamericanos -Argentina, Brasil, Chile, Cuba y México- que mayor representatividad tienen en la producción de las revistas científicas. En cuanto a las áreas científicas elegidas, corresponden a la Astronomía, la Física, la Química, las Matemáticas, las Ciencias de la Tierra, y las de la Ciencia en general.

Para obtener la información sobre los países y áreas científicas antes mencionadas, se acudió a la base de datos - la más ad hoc-Latindex, por ser un sistema de información cuyo propósito es dar visibilidad a las revistas de la región, cuya administración esta a cargo de la Dirección General de Servicios de Cómputo Académico de la UNAM, la misma que proporcionó la información solicitada, para después convertirla en una base de datos, a la que se le denomino LATINTES (2005) con 7.091 registros. Esta incluía todos los títulos vigentes, de todas las disciplinas, y la de otros 47 campos referentes a la revista. Desafortunadamente, no contaba con los campos de temas, títulos indizados e idioma, por lo que tuvo que eliminarse.

Ante ello, permanecía latente la necesidad de contar con registros que incluyeran, además, la temática, indización en fuentes secundarias e idiomas, campos indispensables para el estudio —además de los 47 campos. Dicha información se obtuvo y fue convertida en una base de datos denominada CONSUMOD (2005), conformada por 9.533 registros con la información de los cinco países -Argentina, Brasil, Chile, Cuba y México-, de todas las disciplinas, títulos vigentes. Incluyó los campos de tema(s), títulos indizados -en fuente(s) secundarias- e idioma, de todas las disciplinas, títulos vigentes. Así como los campos de tema(s), título indizados en fuente(s) secundarias - e idioma (estos últimos campos, por el diseño de la base de datos, duplicados o multiplicados).

Con el fin de depurar los registros duplicados y establecer títulos únicos se desarrolló (a partir de CONSUMOD) otra base de datos -BDLATINX-, la cual posteriormente dio origen a dos Bases de datos, las cuales apoyaron decididamente durante todo este estudio. Se trató de DIRLATIN con 659 títulos únicos ubicados en el Directorio Latindex y de CATLATIN con 101 títulos únicos identificados en el Catálogo Latindex.

\section{Las revistas científicas periféricas en América Latina}

La comunicación científica, conforme a Liberman y Wolf (2002), forma parte determinante para explicar y fortalecer a la ciencia misma; tan es así que sin esta comunicación no se podría afirmar que se está realizando efectivamente una actividad científica. Sobre lo anterior, Núñez (2006) puntualiza que la ciencia es una actividad de seres humanos que actúan e interactúan, y por lo tanto una actividad social. Su conocimiento, sus afirmaciones, sus técnicas han sido creados por seres humanos y desarrollados, alimentados y compartidos entre grupos de seres humanos. Por lo tanto, el conocimiento científico es esencialmente conocimiento social.

Durante los últimos años, los niveles de competitividad planteados por la globalización, así como el sitio privilegiado que mantiene la información -y más concretamente la información científica-, vuelven la atención a su producción para crear los medios adecuados a través de los cuales ésta información se evalúe, tratando de garantizar así los niveles de calidad necesarios que son establecidos a nivel internacional, otor- 
gando el reconocimiento y prestigio a los creadores de esta producción.

Entre las prácticas más amparadas para el reconocimiento de la información como producto de investigaciones científicas, se halla la publicación de artículos en revistas especializadas. Para Prieto (1988), el arbitraje de los artículos se encuentra a cargo de organismos y empresas internacionales, como lo es el Institute for Scientific Information (ISI), que se ha destacado en este tipo de evaluaciones, planteándose principalmente proporcionar el reconocimiento de aquellas revistas en donde se expresa la información científica. Aunque cabe contextualizar que dichas evaluaciones presentan información sesgada y con mínima imparcialidad.

Esto permite admitir la presencia de una producción de información científica, a nivel mundial, que robustece los distintos cuerpos teóricos de la ciencia en las diversas disciplinas que la conforman, pero además se hacen de conocimiento público tanto los avances como los resultados para advertir que realmente se esta produciendo ciencia. No obstante, para ser honestos, este tipo de mecanismos de evaluación que se distinguen por la formulación y aplicación de indicadores a los cuales se someten no solo el artículo sino la revista misma - un ejemplo de evaluación a través de la aplicación de indicadores, se puede apreciar en el ensayo de Ríos Ortega (2006) - encuentra una fuerte inclinación hacia lo que se ha denominado el mainstream, el cual tiene la intención de integrar un núcleo principal de revistas y artículos de elite, por llamarlo de alguna manera; donde también destaca la enorme presencia y participación de países altamente industrializados Gaillard (1989) en la elaboración de artículos científicos, que contrasta enormemente con la colaboración y envíos de información que efectúan los países de continentes menos desarrollados, como es el caso de Asia, África y Latinoamérica.

Esta situación da paso a lo que se ha decidido definir como ciencia periférica, la cual implica que la ciencia de los países atrasados es marginal al sistema internacional del conocimiento en términos de recursos y número de investigadores, por mencionar solo algunos de varios factores. Liberman y Wolf (2002), comentan que los científicos en los países subdesarrollados se encuentran limitados en recursos y físicamente lejanos a los centros de asimilación del conocimiento tecnológico, carecen de un sistemático apoyo gubernamental, de tal manera que la difusión de sus resultados conlleva un proceso un poco más prolongado que en otros casos y requiere de un mayor esfuerzo personal de parte del investigador - sin omitir que los medios de comunicación electrónica han facilitado la comunicación entre científicos a nivel nacional e internacional-, y en la calidad y la cantidad de los temas estudiados.

Cueto (1989) alerta sobre la necesidad de subrayar que no toda la ciencia es periférica, y que, por lo tanto, no toda la ciencia de los países atrasados es marginal al acervo mundial del conocimiento, lo que da oportunidad para remarcar que el trabajo científico encuentra en estos países sus propias reglas que deben ser entendidas no solo a partir del atraso o modernidad, sino como parte de su propia cultura y de las interacciones con la ciencia internacional. Al respecto, Núñez (2006) enfatiza revalorizar al nivel de la teoría aquellas prácticas científicas que se desenvuelven fuera de lo que pudiéramos llamar el mainstream, y abrir el camino al estudio de la "ciencia periférica", de la práctica científica que se efectúa en los países subdesarrollados y cuya expresión en términos de número de científicos, publicaciones, patentes y otros indicadores es en realidad modesta al nivel mundial. Así, los estudios de la ciencia han estado tradicionalmente concentrados en los países desarrollados; no obstante, también los subdesarrollados necesitan promover una industria científica capaz de iluminar sus prácticas investigativas, docentes y de políticas científicas, entre otras.

En efecto, si se parte de la idea que conceptualiza a la ciencia en forma universal no podemos dejar de lado las colaboraciones que son producto de una denominada ciencia periférica, y que sus contribuciones forman parte de ese entramado mundial en el que se inserta la ciencia. Por tal motivo, resulta conveniente abordar no solamente la discusión de la ciencia que se desarrolla en países menos desarrollados, sino a su vez avanzar en aquellos productos y/o contribuciones que se observan a través de las llamadas revistas científicas periféricas, las cuales representan un enorme esfuerzo en atención a la productividad de los investigadores regionales, como es el caso del continente Latinoamericano, y de lo que Gaillard (1989) reporta que sí se tiene en cuenta la producción científica total de los investigadores de los países en desarroIlo -aproximadamente $5 \%$ del total de la producción total-, se pone de manifiesto, en efecto, que estos publican en las revistas locales en proporciones aceptables. A su vez, la mayoría de los investigadores publican en revistas locales y en revistas internacionales, variando los países y las disciplinas científicas, así como de aquellos que deciden publicar localmente en idioma propio y lo hacen la mayoría de las veces por elección y no por necesidad. 
En el mismo orden, como objeto de estudio, las revistas científicas periféricas han sido preferentemente estudiadas tanto en el contexto de algunas disciplinas y desde la dimensión de la Bibliotecología (Ríos Ortega, 2006). En ambos casos, la intención ha sido no solo la de establecer una valoración y un reconocimiento de los esfuerzos que se realizan en la creación y publicación de la misma, sino también la de resaltar la situación y el papel que desempeñan para el desarrollo de la ciencia a nivel regional, e igualmente se ha ponderado sobre el número de revistas científicas que sustentan la necesidad de una investigación que de cuenta de su existencia, pero también de una producción. Pero, a pesar de los obstáculos y dificultades, como menciona Vaccarezza (1998), se han sabido sortear para facilitar la continuidad de la publicación de revistas científicas, y con esto visualizar la contribución de los investigadores en la expansión de la ciencia desde un plano regional con miras a un posicionamiento universal.

Para el caso que nos ocupa en el contexto de Latinoamérica, se ha mencionado con insistencia la serie de necesidades con relación a la situación que priva en la producción científica; y, conforme a Velho (2005), existe un considerable acuerdo para acrecentar su aspecto de crecimiento económico. En este entendido se reconoce que Latinoamérica necesita adaptar, comercializar y utilizar el conocimiento, a pesar de esta limitación. La misma autora explica que en la misma región las instituciones académicas del sector público fueron las que produjeron más del ochenta por ciento de las publicaciones durante el año dos mil; $y$, dentro de esta producción, los investigadores se orientaban más -en algunos países- hacia la publicación en revistas del llamado mainstream.

Por los testimonios aquí vertidos, se reconoce la necesidad de abordar la problemática que se presenta en las denominadas publicaciones científicas periféricas producidas en Latinoamérica, en el entendido de que la contribución de este tipo de revistas debe ser revalorada para intentar con esto un posicionamiento distinto al que mantienen hasta el momento. De ahí la trascendencia de nuestra investigación, la cual encuentra como propósito esencial la identificación, cuantificación y trascendencia de las publicaciones ya señaladas para nuestro continente.

En resumen, la atención hacia las publicaciones científicas periféricas resulta ser una inquietud que mueve a considerar la creación de formas y procedimientos que permitan la inclusión de este tipo de publicaciones con la intención de proporcionar una cobertura más amplia a la información contenida en ellas, pero también proporcionar una asistencia de acuerdo a las condiciones y niveles de desarrollo en que se encuentran en sus países de origen.

Asimismo, es necesario reflexionar que, a la par de los envíos realizados por los investigadores a las publicaciones amparadas por el mainstream, es recomendable estimular el envío de sus colaboraciones a las publicaciones periféricas con aquellos investigadores de reciente ingreso; y que esto sirva a su vez de motivación para éstos últimos, quienes podrían avanzar gradualmente en los niveles del mainstream. En todo caso, las colaboraciones en las publicaciones periféricas -a partir de la anterior sugerencia-, no dejan de representar una aportación a la ciencia y, por lo mismo, se vuelve necesaria su integración en espacios similares a los del mainstream, pero bajo características y estándares adecuados a las publicaciones periféricas. En este sentido, la inclusión de este tipo de publicaciones de igual manera representa una forma de contribución para el desarrollo del área científica en los países que aun presentan niveles deficientes en este rubro. De ahí el interés por avanzar en investigaciones que establezcan la posibilidad de identificar en los países con escaso desarrollo científico aquellas publicaciones periféricas cuya aportación resulte significativa para el desarrollo científico nacional e internacional, considerando, por ejemplo aspectos como los siguientes:

1. El acceso y distribución de estar articulado a las instituciones que producen las revistas científicas.

2. Contemplar los canales y mecanismos disponibles - tradicionales o tecnológicos-.

3. El open access como una vía de acceso y distribución al conocimiento científico regional de Latinoamérica.

4. Las vías a considerar para las publicaciones periódicas científicas tienen frente a sí los canales antes mencionados y deben ser entendidos y comprendidos por las instituciones que crean la revista.

\section{Análisis de resultados}

Como ya se indicó en la metodología de esta investigación, se elaboró una cédula de información -misma que puede ser revisada en los anexos-, cuya aplicación permitiera recuperar los datos necesarios de las distintas fuentes de consulta que brindan información sobre las revistas científicas, los países y las áreas del conocimiento a las cuales da cobertura nuestro análisis. La cédula esta integrada por 13 reactivos, por lo que a continuación se exponen los resul- 
tados que se obtuvieron al analizar la información correspondiente. En la Tabla I (en apéndice), se efectúa la cuantificación de las instituciones que realizan investigación científica en los países y áreas del conocimiento seleccionados.

Podemos encontrar que, de acuerdo con los datos proporcionados por diversas fuentes, se logra identificar un número aceptable de instituciones científicas que desarrollan investigación en Latinoamérica -al menos en los países seleccionados-, siendo Cuba, con el 35\% del total, conforme a los datos obtenidos, quien se significa por el mayor número de instituciones; correspondiendo a Brasil (26\%), Argentina $(14 \%)$, México $(13 \%)$ y Chile $(11 \%)$ quienes ocupan del segundo al quinto puesto respectivamente. La escala hace suponer que la tendencia de los países seleccionados con instituciones de representatividad cuantitativa ofrecen un mayor número de publicaciones científicas revistas-, aunque habría que matizar si efectivamente la cantidad de centros de investigación es directamente proporcional a la emisión de revistas científicas, o si en caso contrario el número de instituciones científicas no mantienen un ritmo de productividad elevado en cuanto a dicha producción.

Respecto a la naturaleza de las instituciones científicas que asisten o que contribuyen en la producción y la publicación de revistas en la Tabla II (en apéndice) se detecta que la mayoría de las instituciones científicas se encuentran ubicadas en el sector público. Para quienes han realizado estudios sobre la investigación científica en Latinoamérica - y más específicamente con las instituciones que las respaldan-, este dato ha sido una constante desde años atrás. Para una explicación a este respecto, se pueden formular dos vertientes; la primera es que la pertenencia a este tipo de instituciones - públicas - ha garantizado una fuente de financiamiento para la realización de proyectos, desde un fundamento legal, que el financiamiento es una obligación institucional del Estado; y como una segunda vertiente, cabría advertir los señalamientos que frecuentemente demuestran los bajos recursos económicos que se le asignan a la investigación científica, los cuales, en ocasiones, resultan insuficientes. Así mismo, la relación entre los sectores privados y las instituciones públicas que realizan investigación es una fuente más de financiamiento, a la cual se suele recurrir en momentos que se presentan los adelgazamientos presupuestarios, tan comunes en países latinoamericanos.

Sobre la identificación de la época de creación de las revistas científicas (Tabla III, en apéndice), en Latinoamérica podemos advertir que la presencia de la ciencia es ciertamente joven en nuestro continente con relación al europeo. Esto de alguna forma nos proporciona la orientación adecuada para establecer una explicación con relación a la tradición científica cimentada desde su época de creación, para a su vez identificar si efectivamente este dato corrobora y enriquece la argumentación referente a la afirmación de tendencias hacia determinada área del conocimiento en los países seleccionados. Aunque, en cierto modo resulta adecuado matizar que la tradición científica difícilmente se consolida y conserva en sí misma sin los aportes de recursos que le sean proporcionados a las instituciones donde ésta se desarrolla.

En la identificación de las fuentes de financiamiento en las cuales se soporta la investigación científica (Tabla IV, en apéndice), se vuelve a distinguir la contribución de recursos financieros que se otorgan por parte del sector gubernamental. Esto nos demuestra que la centralización de fuentes de financiamiento por parte de los Estados es un asunto con motivos que favorecen una cortina de humo, ocasionando a veces la búsqueda de fuentes alternativas de financiamiento que pueden ser obtenidas del sector empresarial, por ejemplo, y aún más de aquellos provenientes del plano internacional. Conviene matizar no solo en el cumplimiento de este apoyo, sino incluso profundizar si verdaderamente resulta el más adecuado en función de las recomendaciones que formulan organismos internacionales sobre este respecto.

En atención a la existencia de instituciones gubernamentales responsables de la formulación de políticas científicas, se revela en la Tabla $\mathrm{V}$ (en apéndice) la existencia de instituciones gubernamentales en materia científica en los países latinoamericanos seleccionados; lo que ofrece la garantía de que efectivamente la preocupación y la serie de programas, apoyos legales, acciones e instituciones, indican que la actividad científica es una prioridad dentro de los proyectos de nación en cada uno de estos países. Aunque la fecha de creación de las instituciones no siempre apunta en los mismos años, sí se puede advertir que casi en la mayoría de ellos coincide que es a partir de los años cincuenta y hasta la década de los setenta, cuando no solo se legitiman institucionalmente, sino que vienen a formar parte de una ola de modernización en donde la ciencia aparece como requisito ineludible. Además, se constata que la formulación de políticas científicas necesita obligadamente de una institución que no solo coordine, sino que promueva, estimule y apoye decididamente la actividad científica. 
En tanto que en la cuantificación de la producción de artículos científicos en revistas regionales de Latinoamérica en los países seleccionados en la Tabla VI (en apéndice), la tabla de datos recuperados nos vuelve a ratificar la presencia de Brasil, Argentina y México como países que destacan en la región por su mayor productividad en la elaboración y publicación de artículos científicos. Podemos detectar que durante la década de los noventa aparece un hecho paradójico. Por una parte, se aprecia una leve disminución en cuanto a la productividad en la producción de artículos que comúnmente venían desarrollando Argentina, Brasil, Chile y Cuba. Esto de alguna manera puede encontrar una serie de causas, aunque la más sobresaliente es la referente a que durante la década de los noventa la mayoría de los países latinoamericanos hacen una serie de ajustes a partir de la exigencia de "modernización" que sufren buena parte de las instituciones académicas y de investigación.

Por lo que respecta a la publicación de revistas científicas en los países latinoamericanos seleccionados en las áreas específicas del conocimiento, los datos (Tabla VII, en apéndice) revelan la existencia de un amplio número de revistas cuyos porcentajes parecen indicar la presencia de revistas denominadas periféricas. No obstante, se logra identificar que la mayoría de las revistas responden más a una tendencia de divulgación muy superior a la de aquellas cuyos contenidos de información especializadas destacan de manera primordial.

El fenómeno que igualmente se identificó en el momento de analizar la cuantificación de revistas científicas en los países latinoamericanos aludidos es aquel que se refiere a la distribución de títulos de revistas en una área del conocimiento específica, más bien se integran en una misma revista dos o más temáticas. Se puede deducir que la definición de revistas científicas periféricas encuentra una serie de factores que pueden derivar en una exclusión - de una corriente principal- provocada desde la misma política editorial que deciden las instituciones. Por otra parte, la multiplicación de estas revistas no siempre es sinónimo de corresponder específicamente a una sola especialidad o que, en su defecto, alcancen calificaciones de calidad. Habría que argumentar que la mayoría de la revistas científicas en Latinoamérica no solamente se circunscriben a instituciones de carácter público, sino que su producción se centraliza en las principales capitales de cada país, excluyendo aquellas entidades federativas que cuentan con instituciones de investigación pero cuyos exiguos presupuestos las obligan a la creación de revistas locales que difícilmente logran calificaciones para ser consideradas en una corriente principal.

Respecto a las tendencias en el soporte de la presentación en las revistas científicas en la Tabla VIII (en apéndice) se puede encontrar que, contrario a lo que podría suponerse dentro de la era de la información actual, los datos nos revelan una fuerte inclinación hacia la utilización de la presentación impresa, opuestamente a la reducida utilización de los medios automatizados. Este es, si acaso uno de los rezagos que aun se aprecian en la región; y, por lo mismo, no resulta novedoso el testimonio de que un alto porcentaje de revistas científicas aun continúen en el formato clásico habitual -impreso-, lo cual en cierto modo no resulta del todo negativo. Sin embargo, por otra parte, al no utilizar los medios electrónicos disponibles a nivel mundial, se están perdiendo la oportunidad de interactuar con sus pares de otros países - a través de la utilización de revistas electrónicas. Empero, también debemos reconocer que el porcentaje del 23\% (64 revistas científicas latinoamericanas), es una buena señal que nos induce a considerar que, a la víspera, los orientaciones hacia la utilización de los recursos electrónicos y digitales se está haciendo de manera acertada.

En lo referente a la naturaleza de la publicación científica en los países latinoamericanos, la Tabla IX (en apéndice) muestra que a pesar de que el porcentaje más elevado -79.20 \%refleja que la naturaleza de las revistas alcanzan a ser consideradas precisamente científicas, habría que matizar si efectivamente estas publicaciones cumplen adecuadamente con esta designación a través de la evaluación indicada para este respecto. Esto es, si cumplen con una serie estándares -adecuados a la región y a nivel internacional, por supuesto- 0 si en su defecto la denominación de revistas científicas ha sido utilizada a partir de una práctica heredada o reproducida de otros países. Asimismo, solamente un reducido número de revistas científicas (15) se encuentran ubicadas dentro de una corriente principal, lo cual en buena medida nos hace reconocer que el resto de las revistas científicas no alcanzan a cubrir los estándares planteados por la misma, y por lo tanto no pueden circunscribirse a lo científico; aunque pensar así es ser bastante rigorista y poco flexible, lo cual iría en detrimento de los esfuerzos institucionales realizados en la producción de revistas científicas latinoamericanas.

En relación a las tendencias del idioma utilizado con mayor frecuencia, en la Tabla $X$ (en apéndice) se aprecia que a pesar del dominio del idioma español en la gran mayoría de revistas científicas latinoamericanas, resulta alentador que 
20 de ellas -que representan el $19 \%$ del total de revistas contempladas- estén utilizando el inglés, lo cual mueve a considerar - y no resulta extraño-que las mismas se encuentren en una corriente principal, pero que la utilización de este idioma ha favorecido su incorporación, además, claro esta, del fuerte aporte de una institucionalizada tradición de investigación científica. El idioma viene a ser -en la publicación de revistas científicas - tanto una barrera a nivel internacional como una vía de comunicación a nivel regional.

Respecto a las vías de distribución a las cuales se recurre con mayor incidencia (Tabla XI, en apéndice), se observa que aunque el $33,66 \%$ refiere que no existe información para identificar acertadamente las vías de distribución más frecuentemente utilizadas, todo indica que las vías de distribución más recurrentes $(30.70 \%)$ son la terrestre y la aérea, y por lo mismo, nos hace suponer una fuerte aplicación de recursos económicos en la utilización de las mismas. En contraste, la utilización de recursos alternativos como la Red encuentra niveles de muy baja aceptación y denota que la utilización de la misma no mantiene un desarrollo gradual, y que, en igual sentido, existe una brecha en tecnologías de la información persistente en la región.

En cuanto a la incursión de la revista científica latinoamericana en formato electrónico en la Tabla XII (en apéndice), se puede ver que, a pesar de que los datos pudieran resultar contradictorios respecto a la limitada utilización de las tecnologías de la información en Latinoamérica, lo cierto es que el posicionamiento de Brasil $(21,6 \%)$ en primer lugar, México $(16,08 \%)$ y Argentina $(5,03 \%)$ en segundo y tercer lugares respectivamente en el bienio 2005-2006, da cuenta de un avance gradual y sustantivo de la utilización de formato electrónico de algunas de sus revistas científicas. Una explicación de este hecho pudiera ser el frecuente contacto con instituciones de investigación extranjera, que han contribuido con sus experiencias así como con recursos en áreas de conocimiento donde la colaboración es históricamente reconocida. Pero también destaca la decidida participación de las instituciones responsables de formular políticas científicas, quienes han comprendido ampliamente la necesidad de integrarse en el proceso de globalización del conocimiento científico imperante. Sin embargo, resulta paradójico el caso de Cuba (3.2), donde a pesar de tener una mayor cantidad de centros de investigación, es sumamente débil en la utilización del formato electrónico en comparación con el resto de los países de nuestro estudio.
Por lo que respecta a las revistas científicas latinoamericanas indizadas en los Journal Citation Reports 2005 por países y áreas científicas del conocimiento seleccionadas para el estudio (Tabla XIII, en apéndice), los datos obtenidos para este reactivo vienen a proporcionarnos un panorama no del todo alentador en relación a las revistas científicas de los cinco países latinoamericanos en las seis áreas del conocimiento consideradas. Esto mueve a la reflexión sobre la disparidad entre una cifra y otra, en donde aparece una brecha enorme en relación a la calidad de las revistas que se publican en nuestro continente, y que más propiamente se distingue en los escasos 14 títulos de revistas que logran alcanzar calificaciones aprobatorias en la evaluación que se hace en ISI-JCR. La situación de las revistas científicas latinoamericanas - si bien puede ser valoradas desde aspectos materiales y de contenido de las mismas-, no es sino un mínimo reflejo de una serie de decisiones y acciones vinculadas con la política científica que en ocasiones no alcanzan a dar cumplimiento en la cobertura a los requerimientos que en materia de calidad se demanda de las revistas de nivel internacional en la actualidad. De ahí la necesidad, igualmente, de diseñar y cristalizar acciones que tengan como fin establecer canales de comunicación adecuados que favorezcan la búsqueda de la calidad de las revistas científicas; $y$, también, intentar la creación de un repositorio institucional que a nivel regional integre aquellas revistas científicas que alcancen a mantener estándares de calidad, y, a su vez, dar a conocer aquellas revistas que con frecuencia se producen, pero que son desconocidas incluso en su país de origen.

En cuanto a la distribución por países apreciada en las Bases de datos DIRLATIN y CATLATIN, mismas en las que se apoya este estudio, se puede observar en la Tabla XIV (en apéndice) que en cuanto a DIRLATIN el mayor porcentaje de registros lo tiene Brasil con el $40 \%$, seguido de Argentina con el $26 \%$. Esta distribución, junto con el resto de países, se muestra en la Figura 1. Cabe señalar que en esta base se integran la totalidad de registros localizados en el Directorio de Latindex. Sin embargo, esta distribución cambia notoriamente en CATLATIN donde Argentina con el $27 \%$ encabeza el mayor porcentaje, seguido por Brasil con el $22 \%$, México con el $21 \%$ y Chile con el $20 \%$. Como se puede apreciar en la Figura 2 (en apéndice), los porcentajes entre los países se reducen, lo que indicativo de que los países tiene mayor presencia en el Catálogo de Latindex, donde los títulos han logrado cubrir ciertos parámetros en la evaluación, de lo que infiere la calidad de éstos. 


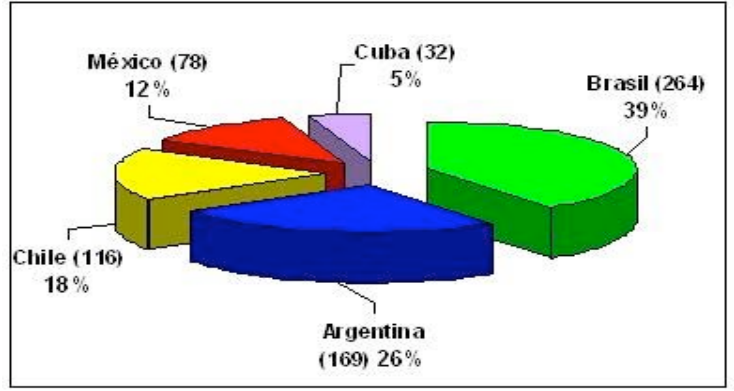

Figura 1. Distribución por países de DIRLATIN

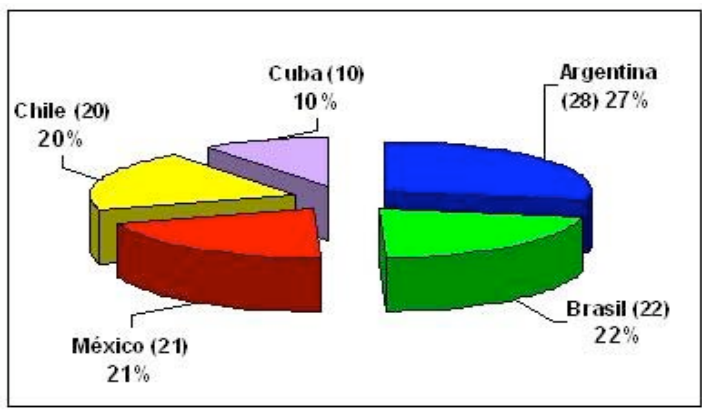

Figura 2. Distribución por países de CATLATIN

\section{Conclusiones}

- En la región latinoamericana se asegura la presencia de las actividades científicas a partir de la existencia de instituciones creadas con este objetivo. Son de carácter público con predominio en instituciones de educación superior, por lo que la producción y publicación de revistas científicas se encuentra centralizada en dichas instituciones y en las principales ciudades.

- La época de creación de la revista científica latinoamericanas no corre paralela a la fecha de fundación de las instituciones que las producen, y sólo un número exiguo llegan a corresponder. La mayoría de las revistas científicas latinoamericanas comienzan a multiplicarse a partir de las décadas de los ochenta y los noventa.

- Las fuentes de financiamiento provienen casi en su mayoría del sector gubernamental y existen instituciones ex profesas para la formulación de políticas científicas, aunque no todos los países mantienen entre sus prioridades la producción y publicación de revistas científicas.

- Las tendencias de las revistas científicas latinoamericanas se inclinan hacia las Ciencias de la tierra y las Ciencias en General —en su conjunto, en ambos casos-y con menores contribuciones en la astronomía, en la que
Brasil, Argentina y México se significan por una participación más elevada en la producción de revistas científicas.

- El soporte de presentación de las revistas científicas es preferentemente en impresión en papel y se emplean mínimamente las tecnologías de la información. Asimismo, la naturaleza de las revistas científicas latinoamericanas alcanza dicha denominación y en menor grado la de técnico profesional, predominando la utilización del idioma español.

- Las vías de distribución continúan siendo la terrestre y la aérea.

- Sólo un reducido número de las mismas alcanzan niveles de calidad conforme a ISI y su JCR. No obstante, el reducido número de revistas científicas que aseguran un sitio dentro de una corriente principal.

- A pesar de que Latindex (Directorio y Catálogo) se ocupa de mantener información actualizada sobre revistas científicas latinoamericanas, sólo se consideran algunos aspectos de las mismas en su parte del Catálogo, pero no así en lo relacionado a los contenidos.

La denominación de revistas científicas periféricas en Latinoamérica se origina desde las características que ofrecen técnica, económica, institucional y naturalmente desde su contenido, más no así desde los esfuerzos de la construcción de la ciencia. A su vez, la integración de la región a las Tecnologías de Información y Comunicación con vistas al acceso y uso de las revistas científicas latinoamericanas debe ser ampliamente impulsado.

\section{Recomendaciones}

- Estimular la creación de un Consejo Regional Científico que atienda la producción científica periférica y que también establezca los mecanismos de difusión de los conocimientos a través de los medios tradicionales y electrónicos disponibles, fortaleciendo las acciones de RICYT en forma conjunta.

- Orientar los esfuerzos por hacer visibles las revistas científicas latinoamericanas en nuevos soportes, entre ellos la Web, y continuar apoyando los esfuerzos como Latindex, Scielo y $D O A J$-entre otros que han demostrado estar a la altura de las circunstancias.

- Vincular las investigaciones de naturaleza cuantitativa-matemática con aquellas de carácter cualitativo, para favorecer así una tendencia de corte multidisciplinario que contribuya a explicaciones y soluciones a medio plazo. 
- Desarrollar cursos de formación para todos los actores involucrados con especial puntualización en la valoración y mejoramiento de las revistas científicas latinoamericanas a la víspera de una posible creación de un Repositorio Continental de estas revistas, con la participación de los bibliotecólogos y/o profesionales de la información.

\section{Referencias}

Allende, Carlos María de (1995). La investigación científica en México. México: Asociación Nacional de Universidades e Instituciones de Educación Superior. 1995. 95

Cueto, Marcos (1989). Excelencia científica en la periferia: actividades científicas e investigación biomédica en el Perú 1890-1950. Lima: GRADE; CONCYTEC, 1989. 230.

Gaillard, Jacques (1989). ¿Es visible la ciencia del tercer mundo? // Mundo científico. 9: 93 (1989) 764-768.

ISI Web of Knowledge. Journal Citation Reports. Science Edition 2005. (2006) http://portal.isiknowledge.com/ portal.cgi?DestApp=JCR\&Func=Frame (2006-02-02)

Latindex. Directorio y Catálogo (2006). http://www.latindex. org/ (2006-01-25).

Liberman, Sofía y Bernardo Wolf. (2002). La comunicación en la formación de grupos científicos. // Memoria del
Simposio Internacional, sobre la información científica: Un enfoque multidisciplinario. Margarita Almada de Ascencio, Sofía Liberman y Jane M. Russell. México: UNAM, CUIB, F.P., 2002. 127.

National Science Foundation (2005). Science and Engineering Indicators 2004. 2. http://www.nsf.gov/statistics/ seind04/pdf_v2.htm (2006-01-23).

Núñez Jover, Jorge. (2006). La ciencia y la tecnología como procesos sociales: lo que la educación científica no debería olvidar. La Habana: Félix Varela. 2006. 245.

Prieto, Fernando E. (1988) El uso de la información científica en México. // Investigación e información científica en México. Ruy Pérez Tamayo coordinador. México: Siglo XXI. 1988. 167.

Red Iberoamericana de Indicadores de Ciencia y Tecnología (RICYT) (1999). Principales Indicadores de Ciencia y Tecnología 1990-1997. Buenos Aires: RICYT, (Junio 1999). http://www.unq.edu.ar/ricyt/ (2006-01-15).

Ríos Ortega, Jaime (2006). Evaluación de normalización de las revistas contenidas en el Indice del Consejo Nacional de Ciencia y Tecnología. // Investigación Bibliotecológica. 20: 40 (enero-junio 2006). 121-148.

Vaccarezza, Leonardo Silvio (1998) Ciencia, tecnología y sociedad: el estado de la cuestión en América Latina. // Revista Iberoamericana de Educación. 18 (1998) 13-40

Velho, Lea (2005) S\&T institutions in Latin America and the Caribbean: an overview. // Science and Public Policy. 32:2 (april 2005) 95-108.

\section{Apéndice}

\begin{tabular}{|c|c|}
\hline Pais & Organizaciones que realizan Ciencia y Tecnologia \\
\hline Argentina & $\begin{array}{l}\text { Educación Superior } 36 \text { universidades públicas y } 43 \text { universidades privadas } \\
\text { Institutos de Investigación Pública en: salud, energía atómica, agricultura, medio ambiente y desarrollo industrial (nivel regional } \\
\text { y nivel nacional), por ejemplo, INTA con } 46 \text { centros de investigación en agricultura distribuidas en } 18 \text { centros regionales. }\end{array}$ \\
\hline Brasil & $\begin{array}{l}\text { Educación Superior: } 150 \text { universidades de investigación (77 públicas y } 76 \text { privadas); } 820 \text { instituciones no universitarias (16\% públicas } \\
\text { y } 84 \% \text { privadas) para la educación profesional. } \\
\text { Institutos de investigación pública y empresas propias del estado: } 22 \text { (1 en salud, } 1 \text { en medio ambiente, } 1 \text { en educación, 1en conocimiento } \\
\text { indigena/Amazónico, } 1 \text { en agricultura, } 2 \text { en salud, petróleo y minas) } \\
15 \text { organizaciones profesionales y nacionales no benéficas (promoción, coordinación y servicios para la agricultura, Pequeñas y } \\
\text { Medias Industrias y capacitación técnica). }\end{array}$ \\
\hline Chile & $\begin{array}{l}\text { Educación Superior: } 67 \text { universidades, } 72 \text { de educación profesional y } 128 \text { de capacitación técnica (23 universidades que llevan a cabo investigación) } \\
\text { Institutos de investigación pública: } 7 \text { (en salud, medio ambiente, conocimiento indigena, agricultura y mineralogía) } \\
\text { ONG's e instituciones profesionales no benéficas (150) } \\
\text { Empresas propias del estado: } 1 \text { (defensa) }\end{array}$ \\
\hline Cuba & $\begin{array}{l}\text { Educación Superior: } 62 \text { universidades públicas (50 universidades están activas en Investigación y Desarrollo) } \\
200 \text { centros de investigación (agricultura y ganado; biotecnología y desarrollo de vacunas y farmacéuticas; saludi; actividades industriales; } \\
\text { plantación de azúcar, biodiversidad y medio ambiente; problemas sociales y económicos) } \\
\text { Ciencia y polos de producción: } 14 \text { redes territoriales. }\end{array}$ \\
\hline México & $\begin{array}{l}\text { Educación Superior. } 1,140 \text { universidades ( } 35 \% \text { públicas y } 65 \% \text { privadas) y } 393 \text { no universitarias (28\% públicas y } 70 \% \text { privadas): la mayoría de } \\
\text { Imvestigación y Desarrollo se concentra en } 3 \text { universidades públicas } \\
\text { Los centros de investigación están vinculados a las universidades públicas } \\
\text { Instituos de investigación pública: } 29 \text { centros SEP-CONACYT }\end{array}$ \\
\hline
\end{tabular}

Tabla I. Cuantificación de las instituciones que realizan investigación científica en los países seleccionados (Fuente: RICYT (2000), apud Velho, 2005, p.95-108). cambiante: el reto de las revistas científicas periféricas latinoamericanas. // Ibersid. (2007) 331-342. ISSN 1888-0967. 


\begin{tabular}{|l|c|c|l|c|c|c|}
$\begin{array}{c}\text { BASE DATOS DIRLATIN } \\
\text { NATURALEZA ORG }\end{array}$ & \multicolumn{7}{c}{$\begin{array}{c}\text { CANT } \\
\text { NASE DATOS CATLATIN }\end{array}$} \\
\hline Institución Educativa & 236 & 35.81 & Institución Educativa & 39 & 38.61 \\
\hline Sin Información & 179 & 27.16 & Asociación científica o profesional & 35 & 34.66 \\
\hline Asociación científica 0 profesional & 93 & 14.11 & Institución de Investigación & 11 & 10.89 \\
\hline Institución Gubernamental & 66 & 10.02 & Sin Información & 6 & 5.94 \\
\hline Institución de Investigación & 53 & 8.04 & Institución Gubernamental & 6 & 5.94 \\
\hline Institución Privada & 30 & 4.55 & Institución Privada & 3 & 2.97 \\
\hline Institución Internacional & 2 & 0.31 & Institución Internacional & 1 & 0.99 \\
\hline \multicolumn{1}{r|r}{ Total } & $\mathbf{6 5 9}$ & $\mathbf{1 0 0}$ & Total & $\mathbf{1 0 1}$ & $\mathbf{1 0 0}$ \\
\hline
\end{tabular}

Tabla II. Naturaleza de las instituciones científicas que asisten o que contribuyen en la producción y la publicación de revistas (Fuentes: Bases de datos DIRLATIN y CATLATIN).

\begin{tabular}{|c|c|c|c|c|c|}
\hline \multicolumn{2}{|c|}{ BASE DATOS DIRLATIN } & \multicolumn{4}{|c|}{ BASE DATOS CATLATIN } \\
\hline ANO INICIO & CANT & $\%$ & ANO INICIO & CANT & $\%$ \\
\hline 1800's & 4 & 0.61 & 1800's & 1 & 0.99 \\
\hline 1900's & 1 & 0.15 & 1920's & 2 & 1.98 \\
\hline 1910's & 1 & 0.15 & 1930's & 2 & 1.98 \\
\hline 1920 's & 5 & 0.76 & 1940's & 6 & 5.94 \\
\hline 1930's & 11 & 1.67 & 1950's & 5 & 4.95 \\
\hline 1940's & 13 & 1.97 & 1960's & 7 & 6.93 \\
\hline 1950's & 24 & 3.64 & 1970's & 8 & 7.92 \\
\hline 1960's & 30 & 4.55 & 1980's & 17 & 16.83 \\
\hline 1970's & 60 & 9.10 & 1990 's & 39 & 38.62 \\
\hline 1980's & 139 & 21.10 & 2000's & 13 & 12.87 \\
\hline 1990 's & 236 & 35.81 & 0 & 1 & 0.99 \\
\hline 2000's & 119 & 18.06 & Total & 101 & 100 \\
\hline 0 & 10 & 1.52 & & & \\
\hline 19 & 3 & 0.46 & & & \\
\hline 197 & 2 & 0.30 & & & \\
\hline 200 & 1 & 0.15 & & & \\
\hline Total & 659 & 100 & & & \\
\hline
\end{tabular}

Tabla III. Identificación de la época de creación de las revistas científicas (Fuentes: Bases de datos DIRLATIN y CATLATIN)

\begin{tabular}{|c|c|c|c|c|c|c|}
\hline \multicolumn{7}{|c|}{ Fuentes de financiamiento para Investigación y Desarrollo en 1996 por porcentaje } \\
\hline Pais & Gobierno & Universidad & Industria & $\begin{array}{l}\text { Sin fines de } \\
\text { lucro }\end{array}$ & $\begin{array}{c}\text { Del } \\
\text { Extranjero }\end{array}$ & Total \% \\
\hline Argentina & 46.3 & 20.3 & 28 & 1.7 & 3.7 & 100 \\
\hline Brasil & 57.2 & 2.8 & 40.1 & NA & NA & 100 \\
\hline Chile & 69.5 & 7.5 & 16.6 & NA & 6.4 & 100 \\
\hline México & 66.2 & 8.4 & 17.6 & 1.1 & 6.7 & 100 \\
\hline
\end{tabular}

Tabla IV. Detección de las fuentes de financiamiento en las cuales se soporta la investigación científica (Fuentes: Red Iberoamericana de Indicadores de Ciencia y Tecnología, 1999. Tomado de: National Science Foundation, 2005).

NA $=$ No Disponible. Notas: Las figuras de las actividades científicas y técnicas de Argentina y Venezuela son más amplias que las de Investigación y Desarrollo que incluyen la capacitación técnica. Los datos de México son de 1995. Los componentes pueden no sumarse al total para evitar discrepancias. cambiante: el reto de las revistas científicas periféricas latinoamericanas. // Ibersid. (2007) 331-342. ISSN 1888-0967. 


\begin{tabular}{|c|c|c|c|}
\hline Pais & $\begin{array}{l}\text { Insituciones encargadas sobre las politicas } \\
\text { en Ciencia y Tecnologia }\end{array}$ & Consejos y Fondos para la Investigación & $\begin{array}{l}\text { Polliticas gubermamentales en el fomento de actividades e } \\
\text { innovación de Investigación y Desamollo en el sector privado }\end{array}$ \\
\hline Argentina & $\begin{array}{l}\text { Secretaría para la Ciencia, Tecnología e Innovación. } \\
\text { (con vimulación al Ministerio de Educación, } \\
\text { Cienciay yecnologia) }\end{array}$ & $\begin{array}{l}\text { Consejo Nacional de Investigación (CONICET } \\
\text { con los tondos ANPCYT, FONTAR, FONCYT) }\end{array}$ & $\begin{array}{l}\text { Ley } 23.877 \text { de } 1990 \text { considera los fondos para la colaboración de } \\
\text { investigación entre los sectores públicos y privados. } \\
\text { Establecimiento de las "units of links" (Unidades de vinculación) } \\
\text { para la identificación, selección y formulación de los programas de } \\
\text { de colaboración en Investigación y Desarrollo. }\end{array}$ \\
\hline Brasil & $\begin{array}{l}\text { Ministerio de Ciencia y Tecnología } \\
\text { Comité principal asesor: Consejo de Ciencia y } \\
\text { Tecnologia (CCT) }\end{array}$ & $\begin{array}{l}\text { Consejo Nacional de Investigación (CNPq), } \\
\text { Financiador de Proyectos (FINEP), } \\
\text { Agencia para la Educación de Egresados } \\
\text { (CAPES); Organizaciones Estatales (FAPs) }\end{array}$ & $\begin{array}{l}\text { Programa en apoyo a la capacidad industrial tecnológica (PCTI) } \\
\text { con varios componenetes: incertivos fiscales para el desarrollo } \\
\text { industrial cientifico y tecnológico (PDTI) y el mismo para la } \\
\text { agricultura (PDTA); apoyo para la innovación tecnológica en las } \\
\text { micro y pequeñas industrias: Proyecto Afa; apoya los proyectos } \\
\text { cooperabivos entre la universidad y la industria: Proyecto Omega; } \\
\text { Programa Nacional para el apoyo de empresas en incubación } \\
\text { (PNI); Programa de Administración Tecnológica para la } \\
\text { Compettividad (PG Tec). } \\
\text { Fondos Sectoriales. }\end{array}$ \\
\hline Chile & $\begin{array}{l}\text { Consejo Nacional de Investigación (vinvulado con } \\
\text { la Oficina Nacional de Planeación (ODEPLA) } \\
\text { que esta vinculada directamerte con el Presiderte } \\
\text { de la República) nivel gubernamental nacional }\end{array}$ & $\begin{array}{l}\text { Consejo Nacional de Investigación } \\
\text { (CONICYT) }\end{array}$ & 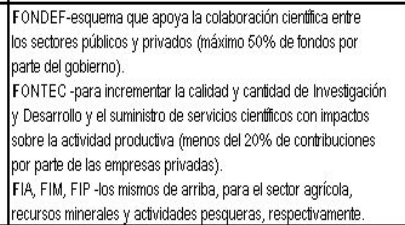 \\
\hline Cuba & Ministerio de Ciencia y Tecnología y Medio Ambierte & Academia Cubana de las Ciencias & Sin información. \\
\hline México & Secretaría de Educcación Pública & $\begin{array}{l}\text { Consejo Nacional de Imvestigación } \\
\text { (CONACYT) }\end{array}$ & $\begin{array}{l}\text { Esquemas que fomenten "espacios del conocimiertb" a nivel regional } \\
\text { irtegrando los instithos de investigación pública con las universidades } \\
\text { locales. Pequeñas y Medias Industias y grandes corporaciones. } \\
\text { Establecimiento de parques de la ciencia. }\end{array}$ \\
\hline
\end{tabular}

Tabla V. Reconocimiento de la existencia de instituciones gubernamentales responsables de la formulación de políticas científicas (Fuentes: Elaborado por la autora de múltiples fuentes: Vonortas (2002); Velho \& Saenz (2002), Velho et al (1998), RICYT (2002), Hansen et al (2002). Tomado de: Velho, 2005)

\begin{tabular}{|l|r|r|r|r|r|r|r|r|r|r|r|r|r|r|r|r|}
\hline $\begin{array}{l}\text { Regióny } \\
\text { paishecononía }\end{array}$ & 1988 & 1989 & 1990 & 1991 & 1992 & 1993 & 1994 & 1995 & 1996 & 1997 & 1998 & 1999 & 2000 & 2001 \\
\hline Argentina & 1.423 & 1.518 & 1.627 & 1.499 & 1.526 & 1.585 & 1.747 & 1.969 & 2.212 & 2.430 & 2.546 & 2.705 & 2.792 & 2.930 \\
\hline Brazil & 1.766 & 2.117 & 2.374 & 2.640 & 3.107 & 2.885 & 3.073 & 3.471 & 3.830 & 4.362 & 5.202 & 5.950 & 6.195 & 7.205 \\
\hline Chile & 682 & 745 & 830 & 819 & 835 & 877 & 798 & 899 & 928 & 974 & 987 & 1.062 & 1.100 & 1.203 \\
\hline Cuba & 67 & 90 & 108 & 94 & 131 & 113 & 141 & 166 & 194 & 177 & 234 & 242 & 282 & 299 \\
\hline Mexic0 & 884 & 1.046 & 1.038 & 1.082 & 1.336 & 1.502 & 1.663 & 1.901 & 2.124 & 2.271 & 2.615 & 2.925 & 2.950 & 3.209 \\
\hline Total & 4.822 & 5.516 & 5.977 & $\mathbf{6 . 1 3 4}$ & $\mathbf{6 . 9 3 5}$ & $\mathbf{6 . 9 6 2}$ & $\mathbf{7 . 4 2 2}$ & $\mathbf{8 . 4 0 6}$ & $\mathbf{9 . 2 8 8}$ & $\mathbf{1 0 . 2 1}$ & $\mathbf{1 1 . 5 8}$ & $\mathbf{1 2 . 8 8 4}$ & $\mathbf{1 3 . 3 1 9}$ & $\mathbf{1 4 . 8 4 6}$ \\
\hline
\end{tabular}

Tabla VI. Cuantificación de la producción de artículos científicos en revistas regionales de Latinoamérica en los países seleccionados (Fuentes: ISI, National Science Foundation, Division of Science Resources Statistics, special tabulations. Obtenido de: Science \& Engineering Indicators 2004.)

\begin{tabular}{|c|c|c|c|c|c|c|c|}
\hline & $\begin{array}{c}\text { CAMPO } \\
\text { CONOCIMIENTO }\end{array}$ & SUBDISCIPLINAS & $\begin{array}{r}\text { DIRECTORIO } \\
\text { LATINDEX }\end{array}$ & $\begin{array}{l}\text { Títulos } \\
\text { ínicos DIR }\end{array}$ & $\begin{array}{r}\text { CATÁlOGO } \\
\text { LATINDEX }\end{array}$ & $\begin{array}{l}\text { Títulos } \\
\text { İnICOS CAT }\end{array}$ & $\begin{array}{l}\text { TíTULOS } \\
\text { JCR }\end{array}$ \\
\hline 1 & Astronomía & & 14 & 14 & 1 & 1 & 1 \\
\hline 2 & Fisica & & 41 & 39 & 7 & 5 & 2 \\
\hline 3 & Química & & 58 & 57 & 22 & 22 & 5 \\
\hline 4 & Hatemáticas & & 84 & 80 & 12 & 12 & 1 \\
\hline 5 & \multirow{8}{*}{$\begin{array}{l}\text { Ciencias de la } \\
\text { Tierra }\end{array}$} & Ciencias de la tierra & 31 & \multirow{8}{*}{238} & 5 & \multirow{8}{*}{33} & \multirow{8}{*}{3} \\
\hline 6 & & Geología ${ }^{*}$ & 90 & & 11 & & \\
\hline 7 & & Geofísica* & 15 & & 3 & & \\
\hline 8 & & Geografía** & 97 & & 15 & & \\
\hline 9 & & Geodesia* & 4 & & 1 & & \\
\hline 10 & & Geoquímica $^{*}$ & 3 & & 0 & & \\
\hline 11 & & Ciencias Atmosféric & 26 & & 4 & & \\
\hline 12 & & Paleontología & 1 & & 1 & & \\
\hline 13 & \multirow{2}{*}{ Ciencias } & Ciencia & 158 & \multirow{2}{*}{231} & 12 & \multirow{2}{*}{28} & \multirow[t]{2}{*}{2} \\
\hline 14 & & Ciencias exact y na & 85 & & 20 & & \\
\hline & Totales & & 707 & 659 & 114 & 101 & 14 \\
\hline
\end{tabular}

Tabla VII. Publicación de revistas científicas en los países latinoamericanos seleccionados en las áreas específicas del conocimiento (Fuentes: Bases de datos DIRLATIN y CATLATIN)***

*Subdisciplinas o especialidades **Disciplina y/o subdisciplina ***Para obtener los títulos únicos del Directorio Latindex, para el estudio (Base de datos DIRLATIN, 659 títulos), fue necesario integrarlos en uno solo, ya que algunos títulos pueden incluirse en dos o más disciplinas. Para obtener los títulos únicos del Catálogo Latindex, para el estudio (Base de datos CATLATIN, 101 títulos), fue necesario integrarlos en uno solo, ya que algunos títulos pueden incluirse en dos o más disciplinas cambiante: el reto de las revistas científicas periféricas latinoamericanas. // Ibersid. (2007) 331-342. ISSN 1888-0967. 


\begin{tabular}{|l|c|c|l|c|c|c|}
\multicolumn{1}{c}{$\begin{array}{c}\text { BASE DATOS DIRLATIN } \\
\text { SOPORTE }\end{array}$} & \multicolumn{7}{c|}{ CANT } & $\%$ & \multicolumn{2}{c|}{$\begin{array}{c}\text { BASE DATOS CATLATIN } \\
\text { SOPORTE }\end{array}$} & \multicolumn{1}{c|}{ CANT } & $\%$ \\
\hline Sin Información & 607 & 92.10 & Impreso en papel & 64 & 63.37 \\
\hline Impreso en papel & 37 & 5.62 & Impreso en papel, En línea & 24 & 23.76 \\
\hline Impreso en papel, En línea & 9 & 1.37 & En linea & 11 & 10.89 \\
\hline En línea & 4 & 0.61 & Disco flexible & 1 & 0.99 \\
\hline CD-ROM & 1 & 0.15 & Impreso en papel, CD-ROM & 1 & 0.99 \\
\hline Impreso en papel, CD-ROM, En línea & 1 & 0.15 & Total & $\mathbf{1 0 1}$ & $\mathbf{1 0 0}$ \\
\hline \multicolumn{1}{|c|}{ Total } & $\mathbf{6 5 9}$ & $\mathbf{1 0 0}$ & & & \\
\hline
\end{tabular}

Tabla VIII. Tendencias en el soporte de la presentación en las revistas científicas (Fuentes: DIRLATIN y CATLATIN).

\begin{tabular}{|c|c|c|c|c|c|}
\hline $\begin{array}{c}\text { BASE DATOS DIRLATIN } \\
\text { NATURALEZA PUBLICACION }\end{array}$ & CANT & $\%$ & $\begin{array}{l}\text { BASE DATOS CATLATIN } \\
\text { MATURALEZA PUBLICACION }\end{array}$ & CANT & $\%$ \\
\hline Revista de Investigación Cientifica & 239 & 36.27 & Revista de Investigación Científica & 80 & 79.20 \\
\hline Sin Información & 205 & 31.10 & Revista Técnico-profesional & 11 & 10.89 \\
\hline Revista Técnico-profesional & 124 & 18.82 & Revista de Divulgación Cientifica y Cultural & 5 & 4.96 \\
\hline Revista de Divulgación Cientifica y Cultural & 70 & 10.63 & Sin información & 4 & 3.96 \\
\hline Técnico-cientifico & 21 & 3.18 & Técnico-científico & 1 & 0.99 \\
\hline Total & 659 & 100 & Total & 101 & 100 \\
\hline
\end{tabular}

Tabla IX. Especificación de la naturaleza de la publicación científica en los países latinoamericanos (Fuentes: Bases de datos DIRLATIN y CATLATIN).

\begin{tabular}{|c|c|c|c|c|c|}
\hline \multicolumn{2}{|c|}{ BASE DATOS DIRLATIN } & \multicolumn{4}{|c|}{ BASE DATOS CATLATIN } \\
\hline IDIOMA & CANT & $\%$ & IDIOMA & CANT & $\%$ \\
\hline Sin Información & 546 & 82.85 & Español & 58 & 57.43 \\
\hline Español & 58 & 8.8 & Español, Inglés & 20 & 19.8 \\
\hline Portugués & 21 & 3.18 & Portugués & 12 & 11.88 \\
\hline Español, Inglés & 17 & 2.58 & Inglés & 5 & 4.95 \\
\hline Inglés & 9 & 1.37 & Portugués, Inglés & 4 & 3.96 \\
\hline Portugués, Inglés & 7 & 1.07 & Español, Inglés, Italiano & 1 & 0.99 \\
\hline Español, Portugués, Inglés & 1 & 0.15 & Español, Portugués & 1 & 0.99 \\
\hline Total & 659 & 100 & Total & 101 & 100 \\
\hline
\end{tabular}

Tabla X. Tendencias del idioma utilizado con mayor frecuencia (Fuentes: Bases de datos DIRLATIN y CATLATIN).

\begin{tabular}{|c|c|c|c|c|c|}
\hline \multicolumn{2}{|c|}{ BASE DATOS DIRLATIN } & \multicolumn{2}{|r|}{ BASE DATOS CATLATIN } & \multirow[b]{2}{*}{ CANT } & \multirow[b]{2}{*}{$\%$} \\
\hline VIAS DE DISTRIBUCION & CANT & $\%$ & VIAS DE DISTRIBUCION & & \\
\hline Sin Información & 411 & 62.37 & Sin Información & 34 & 33.66 \\
\hline Terrestre, Aérea & 83 & 12.59 & Terrestre, Aérea & 31 & 30.70 \\
\hline Terrestre & 69 & 10.47 & Terrestre, Aérea, WMW & 16 & 15.84 \\
\hline WMW & 35 & 5.32 & Terrestre & 6 & 5.94 \\
\hline Terrestre, Aérea, WWW & 22 & 3.34 & WWW & 5 & 4.95 \\
\hline Terrestre, Aérea, Marítima & 14 & 2.13 & Terrestre, Aérea, Marítima & 4 & 3.96 \\
\hline Terrestre, WWW & 11 & 1.67 & Terrestre, WWW & 4 & 3.96 \\
\hline Otras & 6 & 0.91 & Terrestre, Aérea, Marítima, Electrónic & 1 & 0.99 \\
\hline Aérea & 3 & 0.45 & Total & 101 & 100 \\
\hline Terrestre, Electrónica, WMW & 3 & 0.45 & & & \\
\hline Electrónica & 2 & 0.30 & & & \\
\hline Total & 659 & 100 & & & \\
\hline
\end{tabular}

Tabla XI. Vías de distribución a las cuales se recurre con mayor frecuencia (Fuentes: Bases de datos DIRLATIN y CATLATIN). 


\begin{tabular}{|l|c|c|}
\hline Pais & Revistas en texto completo & $\%$ \\
\hline Argentina & 259 & 15.03 \\
\hline Brasil & 372 & 21.6 \\
\hline Chile & 159 & 9.24 \\
\hline Cuba & 55 & 3.2 \\
\hline México & 277 & 16.08 \\
\hline $\begin{array}{l}\text { Otros } \\
\text { Total de revistas en texto } \\
\text { completo }\end{array}$ & 601 & 34.85 \\
\hline
\end{tabular}

Tabla XII. Incursión de la revista científica latinoamericana en formato electrónico (Fuentes: Bases de datos DIRLATIN y CATLATIN).

\begin{tabular}{|c|c|c|c|c|c|c|c|c|}
\hline & & Journal C & Fitation Repo & it 2005, Edició & : Science & & & \\
\hline & Titulo de la revista abreviado & ISSH & Ranking & $\begin{array}{l}\text { Total de Citas } \\
\quad 2005\end{array}$ & $\begin{array}{l}\text { Factor de } \\
\text { Impacto }\end{array}$ & $\begin{array}{l}\text { Indice de } \\
\text { Inmediatez }\end{array}$ & $\begin{array}{c}\text { Artículos } \\
2005\end{array}$ & $\begin{array}{l}\text { Yida } \\
\text { Hedia- } \\
\text { Citas }\end{array}$ \\
\hline 1 & AMEGHINIANA & $0002-7014$ & $29 / 35$ & 711 & 0.481 & 0.191 & 47 & $>10.0$ \\
\hline 2 & AN ACAD BRAS CIENC & $0001-3765$ & $22 / 48$ & 582 & 0.653 & 0.102 & 49 & $>10.0$ \\
\hline 3 & ATM0SFERA & $0187-6236$ & $45 / 47$ & 66 & 0.323 & 0.125 & 16 & \\
\hline 4 & BOL SOC MAT MEX & $1405-213 X$ & $112 / 181$ & 84 & 0.386 & 0 & 11 & \\
\hline 5 & BRAZ J CHEM ENG & $0104-6632$ & $83 / 116$ & 207 & 0.385 & 0.062 & 65 & 5.1 \\
\hline 6 & BRAZ J PHYS & $0103-9733$ & $54 / 69$ & 566 & 0.445 & 0.085 & 212 & 4.8 \\
\hline 7 & J BRAZIL CHEM SOC & $0103-5053$ & $54 / 125$ & 943 & 1.097 & 0.149 & 215 & 4.2 \\
\hline 8 & J CHIL CHEM SOC & $0717-9324$ & $101 / 125$ & 54 & 0.388 & 0.017 & 60 & \\
\hline 9 & LAT AM APPL RES & $0327-0793$ & $92 / 116$ & 99 & 0.301 & 0.083 & 48 & \\
\hline 10 & QUIM NOVA & $0100-4042$ & $82 / 125$ & 876 & 0.65 & 0.119 & 177 & 4.8 \\
\hline 11 & REV CHIL HIST NAT & $0716-078 x$ & 17/24 93/112 & 662 & 0.6 & 0.204 & 49 & 8.1 \\
\hline 12 & REV GE0L CHILE & 0716-0208 & $25 / 36$ & 265 & 0.615 & 0.333 & 15 & 9.5 \\
\hline & REV MEX ASTRON ASTR & 0185-1101 & $15 / 46$ & 565 & 3.234 & 0.059 & 34 & 5.4 \\
\hline & REV MEX FIS & $0035-001 X$ & $66 / 69$ & 200 & 0.123 & 0.053 & 94 & 5.5 \\
\hline
\end{tabular}

Tabla XIII. Cuantificación de revistas científicas latinoamericanas indizadas en los Journal Citation Reports 2005 (ISI; 2006)

Nota: El título Bulletin of the Brazilian Mathematical Society* (1678-7544) tuvo como título anterior: Boletim da Sociedade Brasiliera de Matematica (0100-3569). Es importante señalar que para el estudio se consideró este último, localizado en el Directorio de Latindex; por ello, no se encuentra dentro de los totales de aquellos títulos que figuran con Factor de Impacto para 2005.

\begin{tabular}{|c|c|c|c|c|c|}
\hline \multicolumn{3}{|c|}{ BASE DATOS DIRLATIN } & \multicolumn{3}{|c|}{ BASE DATOS CATLATIN } \\
\hline PAIS & CANT & $\%$ & PAIS & CANT & $\%$ \\
\hline Brasil & 264 & 40.06 & Argentina & 28 & 27.72 \\
\hline Argentina & 169 & 25.64 & Brasil & 22 & 21.78 \\
\hline Chile & 116 & 17.60 & México & 21 & 20.79 \\
\hline México & 78 & 11.84 & Chile & 20 & 19.80 \\
\hline Cuba & 32 & 4.86 & Cuba & 10 & 9.91 \\
\hline Total & 659 & 100 & Total & 101 & 100 \\
\hline
\end{tabular}

Tabla XIV Distribución por países en las Bases de datos estudiadas DIRLATIN y CATLATIN (Fuentes: Bases de datos DIRLATIN y CATLATIN). 